\title{
Determination of thermally stressed state of rod elements of variable cross section under the impact of a lateral heat flow, heat exchange and surface thermal insulation
}

\author{
Sayranbek Akhmetov ${ }^{1 *}$, Anarbay Kudaykulov ${ }^{2 * *}$, and Dossan Bizhanov ${ }^{3 * * *}$. \\ ${ }^{1}$ Atyrau University of Oil and Gas named after Safi Utebayeva, Atyrau, Kazakhstan \\ ${ }^{2}$ Institute of Information and Computing Technologies of the Science Committee of the Ministry of Education and Science of the \\ Republic of Kazakhstan, Almaty, Kazakhstan \\ ${ }^{3}$ JSC "Financial Academy", Nur-Sultan, Kazakhstan
}

\begin{abstract}
A horizontal bar of limited length is considered. The radius of the bar changes linearly along its length. The cross-sectional area of the left end is larger than the right. The lateral surface of the investigated rod is completely insulated. The heat flux is applied to the cross-sectional area of the left end. Heat exchange with the environment takes place through the cross-sectional area of the right end of the rod. The work determines the field of temperature distribution, displacement, three components of deformation and stress, provided that both ends of the rod are rigidly fixed. The amount of bar elongation is also determined when one end of the bar is fixed and the other is free. In case when the two ends of the bar are fixed, the value of the resulting axial compressive force is calculated. In this study the fundamental laws of conservation of energy were used. Key words: Elongation, axial force, section, temperature, displacement, deformation, stress.
\end{abstract}

\section{Introduction}

Many load-bearing elements of gas generating, nuclear and thermal power plants, jet engines and the processing industry are rods of variable cross section. To provide the reliable operation of equipment of those fields, it is necessary to ensure the thermal strength of the load-bearing elements in the form of bars of variable cross-section, which operate under the simultaneous action of different types of heat sources. Due to the variability of the cross section nonlinear thermomechanical processes arise in such rods. To study the nature of such processes a horizontal bar of limited length with variable cross section is considered. In this case, the cross section radius changes linearly along the length of the investigated rod, i.e. $r=a x+b$, $(0 \leq x \leq l)$, where $l$ is the rod length and $a, b$-const. The cross-sectional area of the bar changes nonlinearly along the length of the bar as follows $F(x)=\pi(a x+b)^{2}\left[\mathrm{~cm}^{2}\right]$. The lateral surface of the investigated rod along the entire length is heat insulated. A constant-intensity heat flux $q\left[\frac{\text { watt }}{\mathrm{cm}^{2}}\right]$ is supplied to the cross-sectional area of the left end of the $\operatorname{rod} F(x=0)=\pi b^{2}$. Heat exchange with the environment occurs through the cross-sectional area of the right end of the $\operatorname{rod} F(x=l)=\pi(a l+b)^{2}$. At the same time, the heat transfer coefficient $h\left[\frac{\text { watt }}{\mathrm{cm}^{20} \mathrm{C}}\right]$, the ambient temperature $\mathrm{T}_{\mathrm{a}}\left[{ }^{0} \mathrm{C}\right]$, the physical and mechanical properties of the rod material are characterized by the coefficient of thermal expansion $\alpha\left[\frac{1}{{ }^{0} \mathrm{~K}}\right]$, thermal conductivity $K_{x x}\left[\frac{\mathrm{watt}}{\mathrm{cm} \cdot{ }^{0} \mathrm{~K}}\right]$ and

*Sayranbek Akhmetov: axmetov_aing@mail.ru

**Anarbay Kudaykulov: kudaykulov2006@mail.ru

***Dossan Bizhanov: rector@aogu.edu.kz 
modulus of elasticity $E\left[\frac{\mathrm{kg}}{\mathrm{cm}^{2}}\right]$. The diagram of the investigated rod is shown in Figure 1.

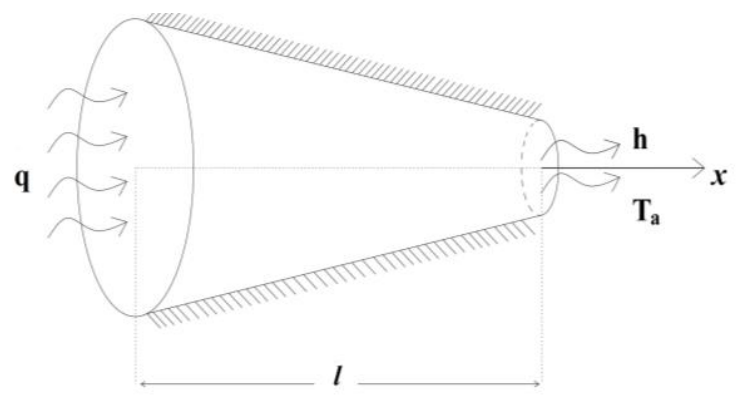

Figure 1: Diagram of the investigated rod.

\section{Overview and Analysis}

In the presence of heat flux, thermal insulation and heat transfer, the functional of the total thermal energy for the investigated rod has the following form [1]:

$$
J=\int_{F(x=0)} q T d s+\int_{V} \frac{K_{x x}}{2}\left(\frac{\partial T}{\partial x}\right)^{2} d v+\int_{F(x=l)} \frac{h}{2}\left(T-T_{a}\right)^{2} d s
$$

where $T=T(x)$ the temperature distribution field along the length of the rod, which is approximated by a full fourth-order polynomial:

$$
\begin{aligned}
& T(x)=a_{0}+a_{1} x+a_{2} x^{2}+a_{3} x^{3}+a_{4} x^{4}= \\
& =\varphi_{i}(x) T_{i}+\varphi_{j}(x) T_{j}+\varphi_{k}(x) T_{k}+\varphi_{m}(x) T_{m}+ \\
& +\varphi_{n}(x) T_{n}
\end{aligned}
$$

where $\varphi(x)$ are spline functions:

$$
\begin{aligned}
& \varphi_{i}(x)=\frac{\left(3 l^{4}-25 l^{3} x+70 l^{2} x^{2}-80 l x^{3}+32 x^{4}\right)}{3 l^{4}}, \\
& \varphi_{j}(x)=\frac{\left(48 l^{3} x-208 l^{2} x^{2}+288 l x^{3}-128 x^{4}\right)}{3 l^{4}}, \\
& \varphi_{k}(x)=\frac{\left(-36 l^{3} x+228 l^{2} x^{2}-384 l x^{3}+192 x^{4}\right)}{3 l^{4}}, \\
& \varphi_{m}(x)=\frac{\left(16 l^{3} x-112 l^{2} x^{2}+224 l x^{3}-128 x^{4}\right)}{3 l^{4}}, \\
& \varphi_{n}(x)=\frac{-3 l^{3} x+22 l^{2} x^{2}-48 l x^{3}+32 x^{4}}{3 l^{4}},
\end{aligned}
$$

$0 \leq x \leq l$ where the nodal temperature values:

$$
\begin{aligned}
& T_{i}=T(x=0) ; T_{j}=T\left(x=\frac{l}{4}\right) ; T_{k}=T\left(x=\frac{l}{2}\right) ; \\
& T_{m}=T\left(x=\frac{3 l}{4}\right) ; T_{n}=T(x=l) ;(4)
\end{aligned}
$$

Considering (2) - (4), minimizing (1) with respect to $T_{i}, T_{j}, T_{k}, T_{m}$ and $T_{n}$, then resolving system of algebraic equations is obtained taking into account the existing natural boundary conditions. By solving the system, the nodal temperature values (4) are determined, and using (2), the temperature distribution field along the length of the rod is constructed. If one end of the rod is rigidly fixed and the other is free, then the elongation of the $\operatorname{rod} \Delta l_{T}[\mathrm{~cm}]$ is determined according to the general law of thermophysics [1]:

$$
\Delta l_{T}=\int_{0}^{l} \alpha T(x) d x .
$$

If both ends of the bar are rigidly fixed, then an axial compressive force $\mathrm{R}(\mathrm{kg})$ arises in the bar, which is determined from the deformation compatibility condition [1]:

$$
R=\frac{\Delta l_{T} \cdot E \int_{0}^{l} F(x) d x}{l^{2}}
$$

In this case a field of distribution of the thermoelastic component of stress $\sigma(x)\left[\frac{\mathrm{kg}}{\mathrm{cm}^{2}}\right]$ appears in the rod:

$$
\sigma(x)=\frac{R}{F(x)}, \quad 0 \leq x \leq l
$$

Then according to Hooke's law it is possible to determine the distribution field of the thermoelastic component of deformations $\varepsilon(\mathrm{x})$ [dimensionless]:

$$
\varepsilon(x)=\frac{\sigma(x)}{E} .
$$

The temperature component of deformations $\varepsilon_{\mathrm{T}}(\mathrm{x})$ is a dimensionless quantity, and is determined according to the general law of thermal physics [1]:

$$
\varepsilon_{T}(x)=-\alpha T(x) .
$$


Then according to Hooke's law the distribution field of the temperature component of the voltage is determined $\sigma_{T}(x)\left[\frac{\mathrm{kg}}{\mathrm{cm}^{2}}\right]$ :

$$
\sigma_{T}(x)=E \cdot \varepsilon_{T}(x)=-\alpha E \cdot T(x) .
$$

According to the theory of thermoelasticity, the laws of distribution of elastic components of deformations $\varepsilon_{x} \quad(x)$ [dimensionless] and stresses $\sigma_{T}(x)\left[\frac{\mathrm{kg}}{\mathrm{cm}^{2}}\right]$ are determined by:

$$
\begin{gathered}
\varepsilon_{x}(x)=\varepsilon(x)-\varepsilon_{T}(x), \\
\sigma_{x}(x)=E \cdot \varepsilon_{x}(x)=\sigma(x)-\sigma_{T}(x) .
\end{gathered}
$$

To determine the displacement field the potential energy of elastic deformations is used [4]:

$$
P E=\int_{V} \frac{\sigma_{x}(x)}{2} \varepsilon_{x}(x) d v-\int_{V} \alpha E \cdot T(x) \cdot \varepsilon_{x}(x) d v .
$$

According to the Cauchy relation [4], it follows:

$$
\begin{gathered}
\varepsilon_{x}(x)=\frac{\partial U}{\partial x} \\
U=U(x)=\varphi_{i}(x) U_{i}+\varphi_{j}(x) U_{j}+ \\
+\varphi_{k}(x) U_{k}+\varphi_{m}(x) U_{m}+\varphi_{n}(x) U_{n},
\end{gathered}
$$

where $\mathrm{U}$ is displacement field.

By minimizing $\mathrm{P}$ by the nodal values of the displacement, a system of linear algebraic equations is constructed. To solve this system it is necessary to set the conditions for fixing the two ends of the bar, i.e. $U_{i}=U(x=0)=0$ : and $U_{n}=U(x=l)=0$.

Further, defining $U_{i}, U_{j}, U_{k}, U_{m}, U_{n} \quad$ and substituting them in (15) the displacement field is constructed. For practical application of the above method and algorithm the following initial data is taken: $l=20 \mathrm{~cm} ; a=-\frac{1}{10} ; \quad b=4 \mathrm{~cm}$; $\alpha=0,0000125 \frac{1}{{ }^{0} K} ; \quad E=2 \cdot 10^{6} \frac{\mathrm{kg}}{\mathrm{cm}^{2}}$ $K_{x x}=100 \frac{w a t t}{\mathrm{~cm} \cdot{ }^{0} \mathrm{~K}} ; \quad h=10 \frac{\text { watt }}{\mathrm{cm}^{2} \cdot{ }^{0} \mathrm{~K}} ; \quad T_{a}=40^{0} \mathrm{C} ;$ $q=-500 \frac{w a t t}{\mathrm{~cm}^{2}}$, where the transition from one temperature unit to another is calculated by the formula ${ }^{\circ} \mathrm{K}={ }^{\circ} \mathrm{C}+273.15$

With these initial data the obtained solution is shown in Figure 2-5.

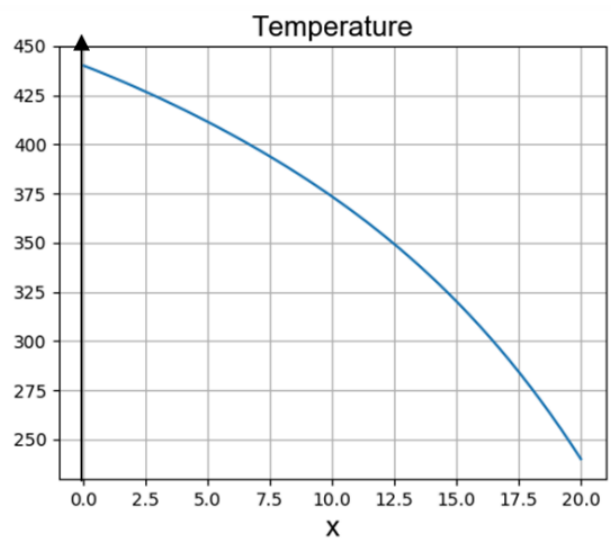

Figure 2: Temperature dependencies along the length of the rod.

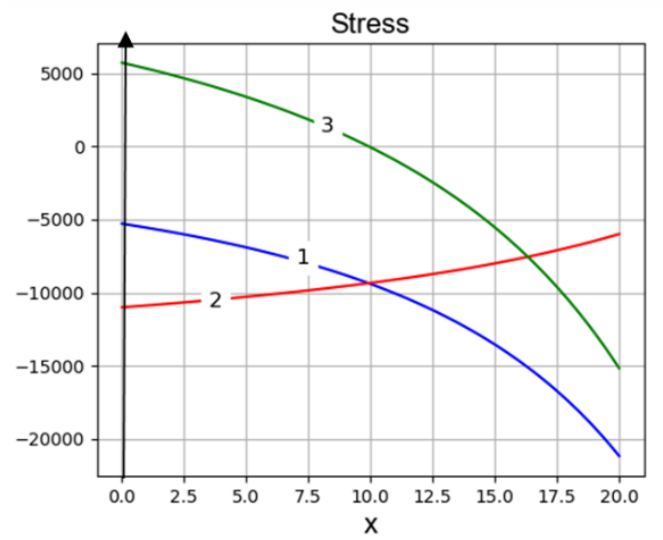

Figure 3: Stress dependencies along the length of the rod.

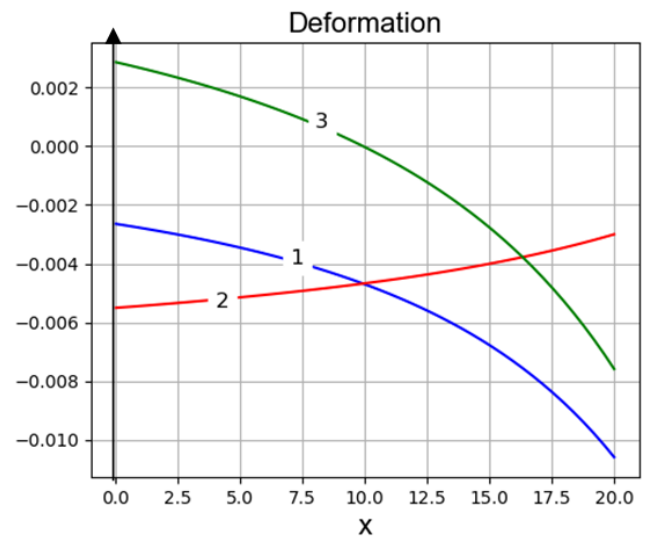

Figure 4: Deformation dependencies along the length of the rod. 


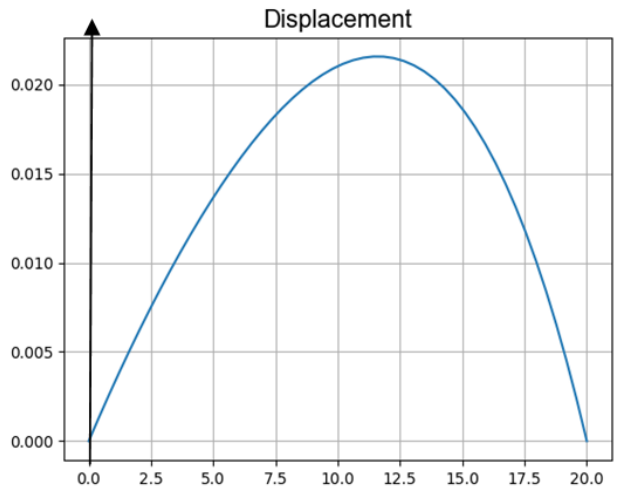

Figure 5: Displacement dependencies along the length of the rod.

\section{Results and discussion}

It can be seen from the Fig. 2 that the temperature value is higher near the left end of the rod, where the heat flux is supplied. Due to the thermal insulation of the lateral surface, heat is lost minimally, as a result of which the temperature at the right end of the rod remains at $240^{\circ} \mathrm{C}$.

The stress dependencies along the length of the rod are shown in Fig. $3(1-\sigma(x)-$ thermoelastic, $2-\sigma_{T}(x)$-temperature, $3-\sigma_{x}(x)$-elastic stress component). Fig. 3 shows that the thermoelastic $-\sigma(x)$ and temperature $-\sigma_{T}(x)$ components of the stress along the entire length of the rod have a compressive character, while the elastic component of the $\operatorname{stress}-\sigma_{x}(x)$ in the region $0 \leq x \leq \frac{l}{2}$ is tensile, and in the region $\frac{l}{2} \leq x \leq l$ it is compressive.

Dependences of deformations along the length of the bar are shown in Fig. $4(1-\sigma(x)$-thermoelastic, $2-\sigma_{T}(x)$-temperature, $3-\sigma_{x}(x)$-elastic component of deformation). The field of distribution of strain components is proportional to the corresponding stresses.

Fig. 5 represents the distribution field of the displacements of the rod fixed at both ends. From this it can be seen that all sections predominantly move in the direction of the $\mathrm{x}$ axis. The greatest amplitude of displacement corresponds to the coordinate $x \approx \frac{3 l}{5}$.

\section{Conclusion}

A numerical model of nonlinear thermomechanical processes in a bar of variable cross-section based on the fundamental law of conservation of energy has been developed. This allows obtaining reliable numerical results considering all-natural boundary conditions. The results obtained are consistent with the corresponding laws of physics. This method can be used to numerically solve a class of problems to determine the steady-state thermomechanical state of load-bearing structural elements operating under the influence of dissimilar types of heat sources, in particular, to study the process of heat transfer and surface insulation in gas turbine systems of thermal power plants.

\section{Reference list}

1. A. Kudaykulov, A. Zhumadillayeva. Numerical simulation of temperature distribution field in beam bulk in the simultaneous presens of heat insulation, heat flux and heat exchange // Acta physica polonica A, pp. 335-336, (2016)

2. Z. Tashenova, E. Nurlybayeva, A. Kudaykulov. Method preparation and solution algorithm for resolving stationary problem of a rod under thermostressed condition restrained at both ends affected by heat exchange and heat flows // International Conference on Advanced Material and Manufacturing Science "ICAMMS 2012". Beijing: pp. 275-277, (2012).

3. N. Muheyat, T. Kuenssaule, A. Kudaykulov. Computer and Mathematical Modeling of Thermomechanical Processes in Element of Constructions // Acta Scientiarum Naturalium Universitatis Nankaiensis. Tianjin: Vol. 50, №3, pp. 56-63, (2017).

4. S. Timoshenko, J.N. Goodyear. Theory of Elasticity / McGRAW-Hill: Company. Inc., 567 p, (1980) 